This item was submitted to Loughborough's Research Repository by the author.

Items in Figshare are protected by copyright, with all rights reserved, unless otherwise indicated.

\title{
A ZigBee-based mobile tracking system through wireless sensor networks
}

PLEASE CITE THE PUBLISHED VERSION

PUBLISHER

(c) Inderscience Enterprises Ltd.

VERSION

AM (Accepted Manuscript)

LICENCE

CC BY-NC-ND 4.0

REPOSITORY RECORD

Alhmiedat, Tareq Ali, and Shuang-Hua Yang. 2019. "A Zigbee-based Mobile Tracking System Through Wireless Sensor Networks”. figshare. https://hdl.handle.net/2134/5768. 
This item was submitted to Loughborough's Institutional Repository (https://dspace.lboro.ac.uk/) by the author and is made available under the following Creative Commons Licence conditions.

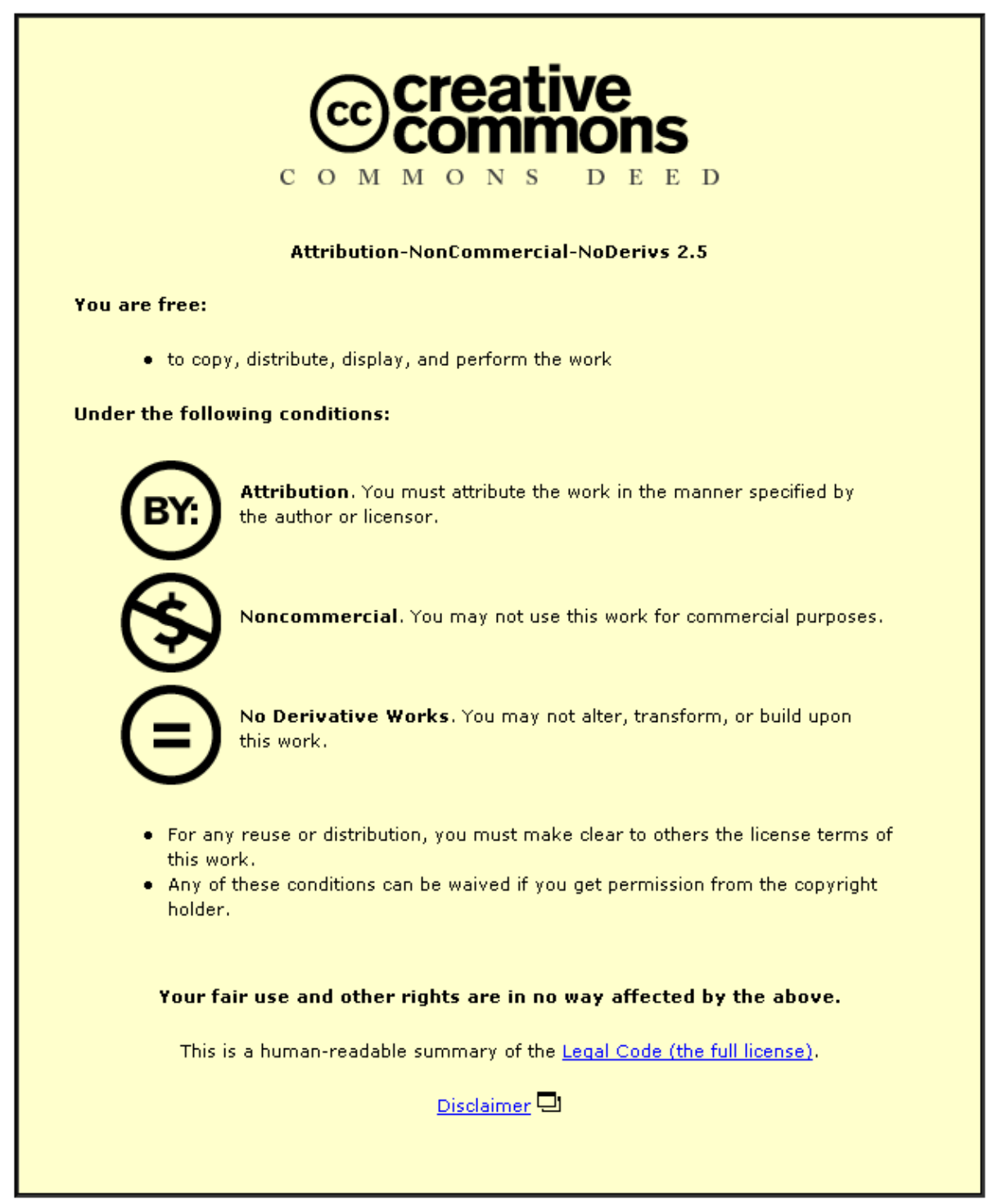

For the full text of this licence, please go to: http://creativecommons.org/licenses/by-nc-nd/2.5/ 


\title{
A ZigBee-based mobile tracking system through wireless sensor networks
}

\section{Tareq Ali Alhmiedat and Shuang-Hua Yang*}

Department of Computer Science,

Loughborough University,

Loughborough LE11 3TU, UK

E-mail: T.Alhmiedat@lboro.ac.uk

E-mail: S.H.Yang@lboro.ac.uk

*Corresponding author

\begin{abstract}
Wireless sensor networks have been deployed widely. Sensor networks involve sensor nodes which are very small in size, low in cost and have a short battery-life. One of the critical wireless sensor network applications is localisation and tracking mobile sensor nodes. ZigBee is a new emerging technology for low rate, low power and low range communication networks, which aims to provide long battery life for network devices. In this paper, we discuss various localisation and tracking techniques and categorise these techniques based on the communication between nodes in centralised and decentralised localisation systems. We propose a decentralised ZigBee-based tracking system to detect and track the location of mobile nodes indoors based on the received signal strength (RSS). The proposed tracking system is a range-free system, which does not require additional hardware, depends on a new weight function, and can be deployed wherever the node density is low. The tracking system is implemented by ZigBee sensor devices, and experiments are done to evaluate the proposed tracking system based on accuracy and communication cost.
\end{abstract}

Keywords: localisation; tracking.

Reference to this paper should be made as follows: Alhmiedat, T.A. and Yang, S-H. (2008) 'A ZigBee-based mobile tracking system through wireless sensor networks', Int. J. Advanced Mechatronic Systems, Vol. 1, No. 1, pp.63-70.

Biographical notes: Tareq Alhmiedat is a PhD student in the Computer Science Department at Loughborough University. He received his MSc from University of the West of England, UK, in 2005 and BSc from Applied Science University in 2004, Jordan. His research interests include tracking mobile targets through distributed sensor networks.

Shuang-Hua Yang is a Professor of Networks and Control. He is the Director of the Networks and Control Research Group in the Computer Science Department at Loughborough University. He is also an overseas Professor in Central China Normal University and a Guest Professor in Huazhong University of Science and Technology, Petroleum University China and Liaoning University of Petroleum and Chemical Technology. His research interests include wireless sensor networks, networked control, safety critical systems and real time software maintenance. He is a Fellow of the Institute of Measurement and Control (FInstMC), a Senior Member of IEEE (SMIEEE) and a Chartered Engineer (CEng) in the UK. He is an Associate Editor of the International Journal of Systems Science and the International Journal of Automation and Computing, and a member of the Editorial Advisory Board for other four international journals.

\section{Introduction}

Wireless sensor networks have become a vital research area nowadays and sensor nodes are used widely. The first research in this area was motivated by military applications with DARPA funding a number of prominent research projects such as smart dust and NEST. Recently, civilian applications for wireless sensor networks have been considered, including environment and species monitoring, water, air, soil chemistry, agriculture, production and delivery and healthcare. Wireless sensor networks are composed of sensor nodes, which collaborate to perform specific tasks. Sensor nodes have the ability to sense, process, and communicate data. The main goal of wireless sensor networks is to permit multiple applications to run on top of the same sensor network.

Sensor networks are a system of many small and simple devices deployed over an area in order to sense and monitor events of interests or track objects or people as they move. As shown in Figure 1, sensor nodes are tiny electronic devices equipped with a battery for an energy source. They have a sensor for detecting physical characteristics and a processor for performing computations. A wireless transceiver is fitted for two way communications with other 
sensors. They are equipped with a memory for storing information. A sensor node has the following characteristics:

1 small physical size

2 low power consumption

3 limited processing power

4 short-range communications

5 a small amount of memory storage.

Figure 1 Wireless sensor (Jennic) (see online version for colours)

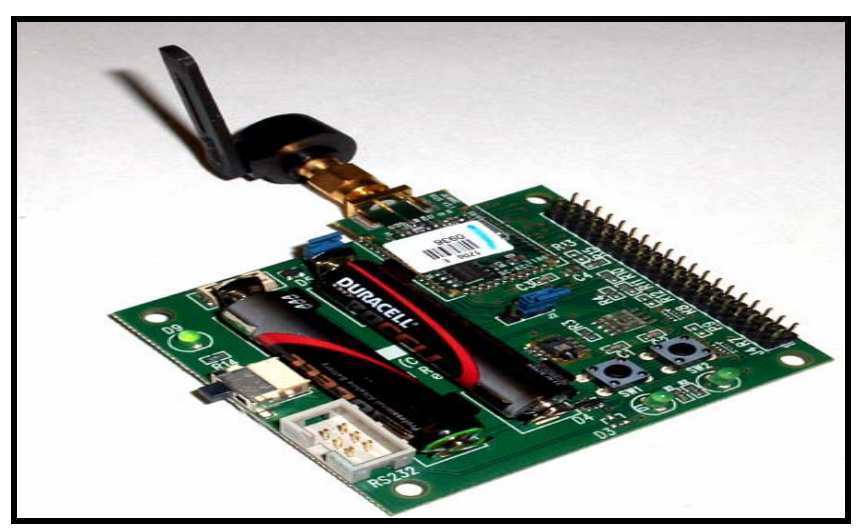

Localising wireless sensors and tracking mobile targets through wireless sensor networks have become two important areas in the use of wireless sensor networks. Localisation involves determining the location of the sensor node based on other sensor nodes with known locations. Tracking mobile targets involves finding out the location of mobile targets based on wireless sensor nodes with known positions. According to Shorey et al. (2006), target tracking using wireless sensor networks was initially investigated in 2002. In this paper, we concentrate on tracking mobile targets using sensor nodes within fixed locations. The main problem is detecting the presence of mobile targets based on the distributed sensor nodes without using any additional hardware. The technique must be inexpensive and power efficient.

The global positioning system (GPS) is the most widespread outdoor positioning system for mobile devices. GPS provides the locations for mobile devices with high accuracy worldwide, based on 24 satellites and three redundant backups. A GPS system can not be deployed with wireless sensor devices for the following reasons: Cost: attaching a GPS receiver with hundreds or thousands of sensor nodes is not a cost-effective solution. Limited power: each sensor device has a limited amount of power, and a GPS receiver requires high amounts. Inaccessibility: GPS receivers do not work indoors, which is one of the main drawbacks. Form factor: the size of GPS receiver is too big if it's compared to a sensor device. Consequently, GPS is not the ideal approach for localising and tracking purposes in wireless sensor networks.

This paper is organised as follows. Section 2 reviews the existing localisation and tracking techniques. Section 3 presents a weighted LQI system model and our tracking phases. Section 4 involves implementing our work on real ZigBee-based sensor devices. Section 5 includes evaluating the presented approach based on accuracy and communication cost. And finally, Section 7 gives a conclusion and future works.

\section{Localisation and tracking systems}

Localisation and tracking systems have been deployed widely in many applications, such as military, civil and forest monitoring applications. Military applications include tracking soldiers and tanks in the field. Civil applications involve monitoring people and materials. And finally, forest applications involve tracking animals or birds in a forest field.

Localisation systems can be divided based on the communications between nodes, into centralised and decentralised systems. Centralised systems involve transmitting all the localisation information to a central computer in order to calculate and find out the positions for the target nodes. Decentralised systems depend on each sensor node to calculate its position with only limited communication with nearby nodes, and hence there are no centralised computations and communications.

Centralised localisation information requires sending all the localisation information to a central node, which is quite expensive, since the power supply for each node is limited. Consequently, communication with centralised computing is a quite expensive localisation system, and sending time series within the network introduces latency, in addition to consuming energy and network bandwidth. There are many centralised and decentralised localisation techniques described by Alhmiedat and Yang (2007).

\subsection{Received signal strength}

Received signal strength (RSS) systems have been deployed widely in many localisation applications. RSS systems involve finding out the location of the target nodes based on measuring the RSS values from several stationary sensor nodes with known positions. The main concept behind RSS system is that the configured transmission power $P_{T}$ at the transmitting device can directly affect the received power $P_{R}$ at the receiving device. Based on Rappaport (1996), in free space transmission model, the detected signal strength value decreases with the distance to the sender.

$$
P_{R}=P_{T} \times G_{T} \times G_{R}\left(\frac{\lambda}{4 \pi d}\right)^{2}
$$

where, $G_{T}, G_{R}$ are gain of transmitter and gain of receiver respectively. $\lambda$ is a wave length, and $d$ is the distance between sender and receiver. The received signal strength is converted to a received signal strength indicator (RSSI) which can be defined as ratio of the received power to the reference power $P_{\operatorname{Re} f}$. 


$$
R S S I=10 . \log \frac{P_{R}}{P_{\text {Re } f}}
$$

RSS-maps localisation system is a common approach based on the RSS technique. This system involves measuring the received signal strength at particular locations; it assumes that each position inside a building has a unique RF signature. Brunato and Battiti (2005) and Roos et al. (2002) propose localisation techniques which involve measuring the signal strength in passive mode from several access points and then storing it in database. These are power-consuming and complicated techniques, because exhaustive data collection is needed for a wider area network.

\subsection{Link quality indicator}

Link quality indicator (LQI) represents the quality of the connection between sender and receiver. LQI measurement involves the characterisation of strength and/or quality for each received packet, and the results should be reported as an integer ranging from 0x00 to 0xff. The strongest LQI value means the best connection quality between sender and receiver, and the lowest LQI value means the minimum quality of signal which is detected by a receiver.

\subsection{ZigBee network standard}

ZigBee is a low power, low rate, low cost wireless communication standard, which aims to be used in home automation and remote control applications. ZigBee standard has been designed to offer minimum cost and power connectivity for devices which require battery life for durations ranging from several months to several years. ZigBee devices are expected to cover 10-75 meters based on the RF environment and output consumption required for a given application.

Each ZigBee network involves three main components as shown in Figure 2: coordinator (ZC), routers (ZR) and end-devices (ZED). Only one coordinator is required for each ZigBee network, and it initiates the network formation. A router is an optional network component. It may associate with coordinator, and participates in the multi-hop routing of messages. And finally, an end-device which is optimised for low power-operation and only connects to one coordinator or router.

Figure 2 ZigBee mesh network (see online version for colours)

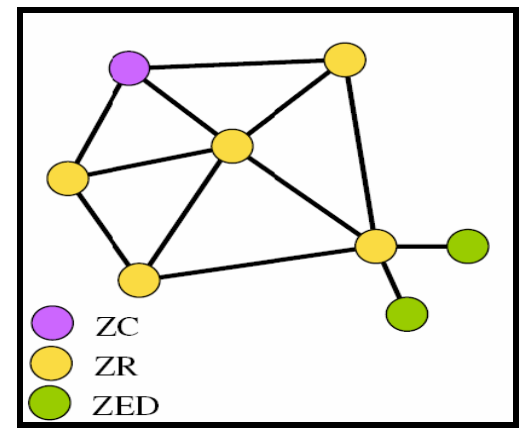

The most important advantage behind deploying our localisation technique with ZigBee standard is the simplified implementation process with the provided protocol suit of ZigBee. Moreover, the low complexity, fast calculation, and the minimum resource requirements, make ZigBee standard an ideal network solution for wireless sensor nodes.

\section{Weighted LQI system model}

Time of arrival (TOA) and time difference of arrival (TDOA) techniques have been deployed in many localisation systems, since they offer high localisation information. These techniques are not ideal localisation solutions for cheap sensor devices as they require additional hardware to be attached to each single node, consequently, increasing the sensor's complexity and cost.

RSS is a cheap and simple localisation solution for outdoor environments. It gives accurate localisation information. Deploying a RSS system in indoor environments is extremely difficult because of the obstacles in the propagation environment. These environments affect RSS seriously by either enhancing or dispersing it.

Many previous works adopt a new weight function to increase the position accuracy of the target nodes. This solves the problems of RSS not offering highly accurate position information. The weight function depends on the distance and the environmental characteristics between sender and receiver.

We believe that the weight function can be measured in many different ways. There are several related works which have used the weights to measure the distance between beacon nodes and stationary sensor nodes.

\subsection{Related work}

In this section, we present the works which related directly to our work. The presented work in Bulusu et al. (2000) involves a localisation technique based on connectivity metric and radio frequency. This technique depends on a spherical radio propagation assumption. The proposed system works efficiently in outdoor environments, and in their future work they propose to adapt this localisation system to noisy environments. In Blumenthal et al. (2005), a new localisation solution to reduce the error of the weighted centroid localisation algorithm, which depends on hop count determination. It is assumed that the transmission range for each sensor is represented as a single circle.

Blumenthal et al. (2007) propose a ZigBee weighted centroid localisation algorithm to locate devices with unknown positions in wireless sensor networks. The proposed system works efficiently in outdoor environments, and is tested using ZigBee-based sensor devices (four routers and one coordinator). The coordinator is considered to be a mobile target and the beacon nodes as routers. Reichenbach et al. (2006), proposed novel optimisations for coarse grained localisation systems with centroid determination to find out the position of the target nodes in 
a precise way. This work is similar to the work presented in Blumenthal et al. (2005). Both works focus on computing an optimal transmission range for all beacon nodes to reduce total energy consumption.

Work in Reichenback and Timmermann (2006) is similar to the work introduced in this paper. They use a weighted centroid localisation algorithm in combination with RSS at indoor environments. The proposed system involves using methods like frequency diversity and averaging multiple measuring data in order to reduce the localisation error. They achieved a small localisation error of $14 \%$ for $69 \%$ of all test points. However, they deploy their work in a small experimental area $(300 \mathrm{~cm} \times 300 \mathrm{~cm})$.

\subsection{Our tracking approach}

Several tracking systems in previous works were deployed for several purposes. In our research, we concentrate on tracking mobile targets through distributed low density sensor nodes. A new approach is used to improve the localisation accuracy for sensor nodes based on a weight function. We believe that the weight function can be implemented in different ways. Such as in Bulusu et al. (2000), where the weight is based on the difference between the sending messages and received acknowledgements.

Our approach calculates the weights between beacon nodes with known positions, based on finding out the relationship between distances and RSS values. We assume that the stationary sensor nodes are with known positions within a building layout and the layout of this building is known. The proposed tracking system involves three main phases as shown in Figure 3.

Figure 3 Tracking phases (see online version for colours)

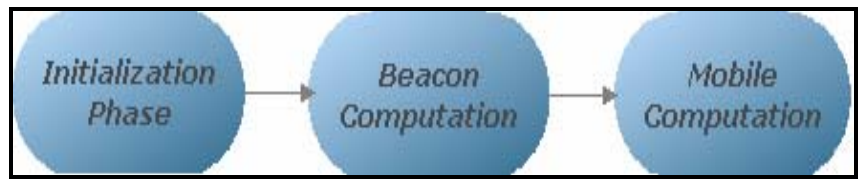

\subsubsection{Initialisation phase}

The mobile target sends 'hello' messages to all beacon nodes in its area, and waits for responses from them. All sensor routers with known positions in the mobile target's area send acknowledgements to the mobile target. The mobile target selects at least three stationary beacon nodes, to be used in the localisation process. If the mobile device receives less than three acknowledgements, it sends the 'hello' message again. This phase is repeated until receiving acknowledgments from three different beacon nodes.

\subsubsection{Beacon nodes computation phase}

In this phase, weights are calculated based on the selected beacon nodes. The weight $w_{i j}$ can be measured between each beacon nodes pair $B_{i}$ and $B_{j}$. For instance, if the mobile target is covered by three beacon nodes, there will be three different weights between these nodes as shown in Figure 4. The weights are calculated based on the distance between beacon nodes and RSS values. It is important to note that distances between beacon nodes are known and fixed. $\operatorname{Dist}\left(B_{i}, B_{j}\right)$ refers to the distance between stationary nodes $B_{i}$ and $B_{j}$. For simplicity, the computed weight is multiplied by 100 . The weight $w_{i j}$ is calculated between nodes $B_{i}$ and $B_{j}$ as follows:

$$
w_{i j}=\left(\frac{\operatorname{Dist}\left(B_{i}, B_{j}\right)}{\operatorname{RSS}\left(B_{i}, B_{j}\right)}\right) \times 100
$$

The $R S S\left(B_{i}, B_{j}\right)$ can measure the signal strength value between $B_{i}$ and $B_{j}$, through measuring the signal strength value for each packet received. The average weight $A v e_{w}$ can be introduced as the main characteristics for the main environment. It can be represented in equation 4 .

$$
A v e_{w}=\frac{\sum_{i=1}^{n} w_{i}}{n}
$$

The mobile target can find out its position based on the received weights and the RSS from different beacon nodes.

Figure 4 Weighted approach (see online version for colours)

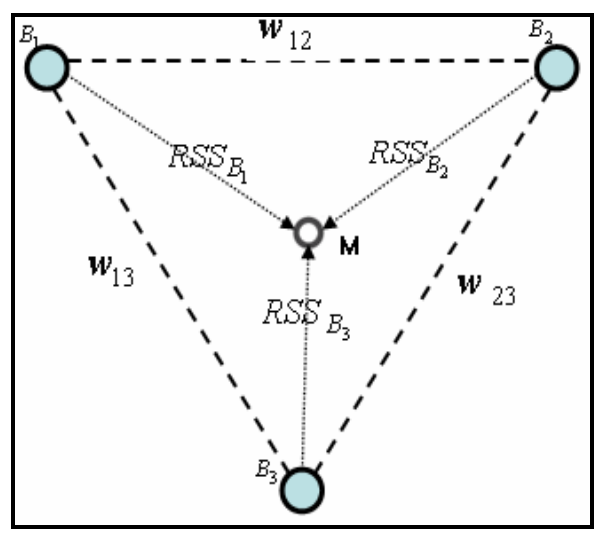

\subsubsection{Mobile computation phase}

Each mobile node receives at least three different weights from beacon nodes, in addition to the RSS values for each stationary beacon node. The mobile node can improve the localisation accuracy based on adjusting the received signal values from several beacon nodes. Calculating the final distance between target node and beacon nodes is executed in the mobile target device. This is due to the mobile target device's larger memory size, faster processor and longer battery life. The mobile target can be a laptop or PDA device, and therefore can display the current position for the mobile target. 
Figure 5 Tracking algorithm

Begin

1 Initialisation phase:

1.1 For each mobile target $M_{i}$

1.1.1 Send 'HELLO' messages to all beacon nodes $B_{i}$ in its area.

1.1.2 Wait responses from beacon node.

1.2 For each beacon node $B_{i}$ : send responses to each mobile node $M_{i}$

Repeat till getting responses from 3 different beacon nodes

2 Beacon computation phase:

2.1 Compute weights $w_{i}$ for each pair of nodes

If $i<3$ then

Go to initialisation phase

else send weights to each beacon nodes

End if

2.2 Send the computed weights $\sum w_{i}$ to each mobile target in their transmission range

3.3 Find out the overall environment characteristics based on equation 4

3 Mobile computation phase:

3.1 For each mobile target $M_{i}$, weights and RSS values are collected from each beacon node. For each $B_{i}$ in mobile target transmission range: collect the RSS values and weights from all beacon nodes.

3.2 Each mobile target calculates the distance between itself and each beacon node based on the received weights and signal strength values, as shown in equation 5.

3.3 A triangulation technique is applied in order to measure the final position for a mobile target.

End

Target node improves its location accuracy by comparing the RSS values and the received weights. If the mobile target is covered by three beacon nodes, then every signal strength value received by a beacon node can be adjusted based on two different weights $w_{i j}$ and $w_{i j \pm 1}$. Two distances will be calculated based on the measured weights; the final distance will be calculated based on averaging both measured distances. This phase is repeated for all the RSS values. As shown in Figure 4, there are three beacon nodes, therefore, each beacon node is connected with two beacon nodes, and two weight values can be measured $w_{i j}$ and $w_{i j \pm 1}$. In this case, the mobile target can calculate the distance between itself and each beacon node based on the following equation:

$$
\operatorname{Dist}\left(M, B_{i}\right)=\frac{\left(R S S\left(M, B_{i}\right) \times w_{i j}\right)+\left(R S S\left(M, B_{i}\right) \times w_{i j \pm 1}\right)}{n-1}
$$

where $n$ is the total number of beacon nodes, $\operatorname{Dist}\left(M, B_{i}\right)$ refers to the distance between mobile node $M$ and beacon node $B_{i}, \quad j=\{1,2,3\}$ depends on the number of beacon nodes which are connected to beacon node $B_{i}$. In this case, three different distances $M B$ are measured based on the previous equation as the mobile target connects to at least three beacon nodes. A triangulation technique is applied in order to find out the final location of the target node $M$. A triangulation method involves determining the relative positions of points in space by measuring distances between points with unknown positions and reference points. The tracking algorithm is summarised in Figure 5.

\section{Experiments}

Our tracking system can be deployed in many indoors applications, such as tracking patients in hospitals and fire-fighters in emergency situations.

In this section, we explain the main features of sensor devices which have been used in our experiments. The building's layout is depicted in Figure 10, which shows positions of beacon nodes. RSS values have been collected in several mobile's device positions from three different beacon nodes. Finally, the collected RSS values were improved based on the measured weights from several beacon nodes.

\subsection{Hardware platform}

In our experiments, we use JN5139-EK010 sensor node platform. This module offers low power consumption, low processor overhead and a low cost platform for wireless sensor networks. It supports complex tree or mesh network topologies providing reliable coverage over large areas. Jennic's ZigBee stack API offers rapid application development by providing simple programming to the standard ZigBee network layer. Figure 1 shows a sensor node from Jennic.

Usually, ZigBee network standard involves three different types of devices: coordinator, routers and end-devices.

\subsection{Experimental testbed}

Our experimental testbed involves four main sensor nodes. One of them is used as a receiver and the rest are used as beacon nodes with known positions. There are two main sensor devices that are used in our experiments:

1 Coordinator: there is only one coordinator in the network. It's looked upon as a mobile target in our experiments. It is responsible for collecting beacon 
packets from several stationary sensor nodes in addition to collecting the weights. The received packets are transferred to a laptop connected with the coordinator in order to calculate the position of the mobile target node.

2 Router: there are three router devices with known positions. Routers are reference devices, and they send beacon packets to every mobile target in their range. Each router can calculate weights between itself and the other routers in its range.

We have done our experiments at Loughborough University, in the FK research area $(41.5 \times 11.3 \mathrm{~m})$. Figure 10 shows the main structure of FK area. Three router devices (beacon nodes) were deployed in that area, as indicated in the figure by the black dots. This area involves nine offices and two meeting rooms. The rest contains desks, cabinets and chairs which affect the RSS values.

\subsection{Analysing RSS-measurements}

To grasp the relationship between distance and RSS values in an indoors environment, RSS values were gathered from three beacon nodes in FK area. We deployed three beacon nodes in three different positions, and collected the RSS values between each one and the mobile target in several points. The RSS values were collected on the distances (0.5, 1, 2, 4, 8, 12, 16, 20, 24, 28, 32m), as seen in Figure 6. This process was repeated 12 times, each called a treatment.

Figure 6 Analyzing RSS values through different points (see online version for colours)

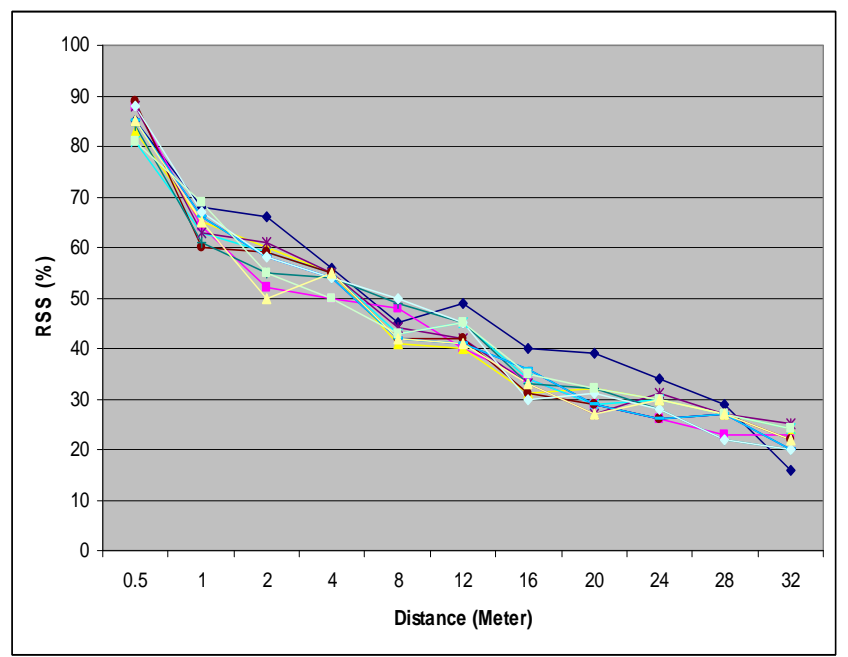

We can notice that the RSS values are affected by obstacles, and hence reduce or increase the signal values. As in Figure 6 , there are no significant differences between signal strength values collected for different treatments on several different distances. This indicates that distance affect the value of RSS. Consequently, it's possible to depend on the RSS to measure the distance. Figure 7 shows the average of RSS values through several distances.
Figure 7 Averaging RSS values (see online version for colours)

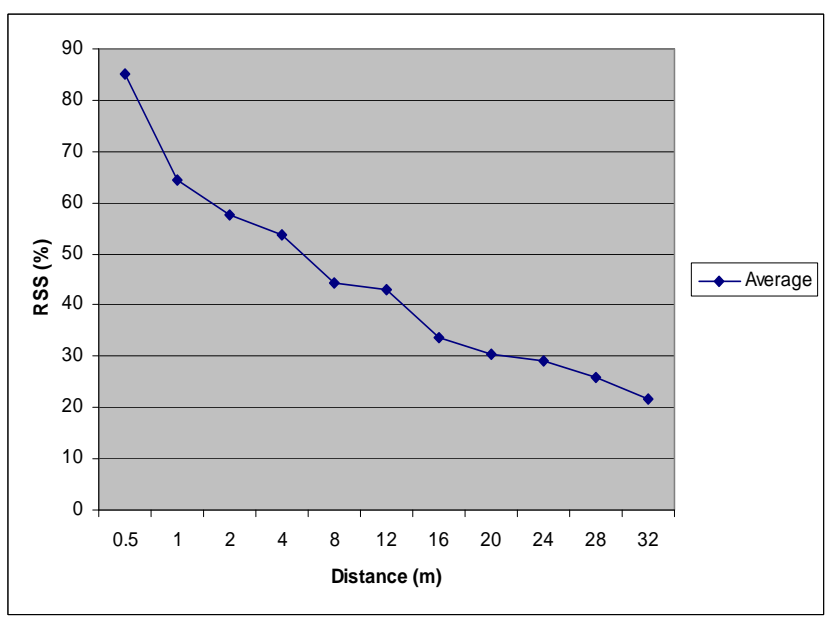

\subsection{Adjusting RSS values}

RSS values are usually affected by obstacles and walls; hence, finding out the location of the target nodes based on signal strengths has some limitations. The RSS values should be analysed and processed in order to get efficient localisation information. In our approach, RSS values are updated based on the computed weights from beacon nodes in the mobile target's area. Weight function is based on the RSS and distance between each pair of beacon nodes.

The measured received signal values are adjusted by applying a weight function to each measured received signal value, as shown in equation 5 . By this process, it is possible to get RSS values closer to those that would have been attained if the environment characteristics were ideal. This also means that the effect of boundaries will be almost eliminated. Based on that, the final distance between each beacon node and mobile target can be calculated using equation 5 .

\section{Evaluation}

In this section, we evaluate our weighted tracking approach by evaluating the effect on accuracy and communication cost. Results are compared with previous works.

\subsection{Accuracy}

The localisation error $E$ can be found based on the distance difference between actual position $P_{i}\left(x_{\text {actual }}, y_{\text {actual }}\right)$ and approximated position $P_{i}\left(x_{\text {new }}, y_{\text {new }}\right)$ of mobile node $i$ :

$$
E_{i}=\sqrt{\left(x_{\text {actual }}-x_{\text {new }}\right)^{2}+\left(y_{\text {actual }}-y_{\text {new }}\right)^{2}}
$$

Figure 8 shows the localisation error in meters through several points. Results show that localisation error is between 2 and 6 meters. We measured the target's position in several points in FK area. As mentioned, the FK area involves many obstacles and walls which can affect the RSS and hence affect the localisation accuracy. The weight 
function has been used to minimise the localisation error, and results are shown in Section 5.3.

Figure 8 Localisation errors in weighted approach (see online version for colours)

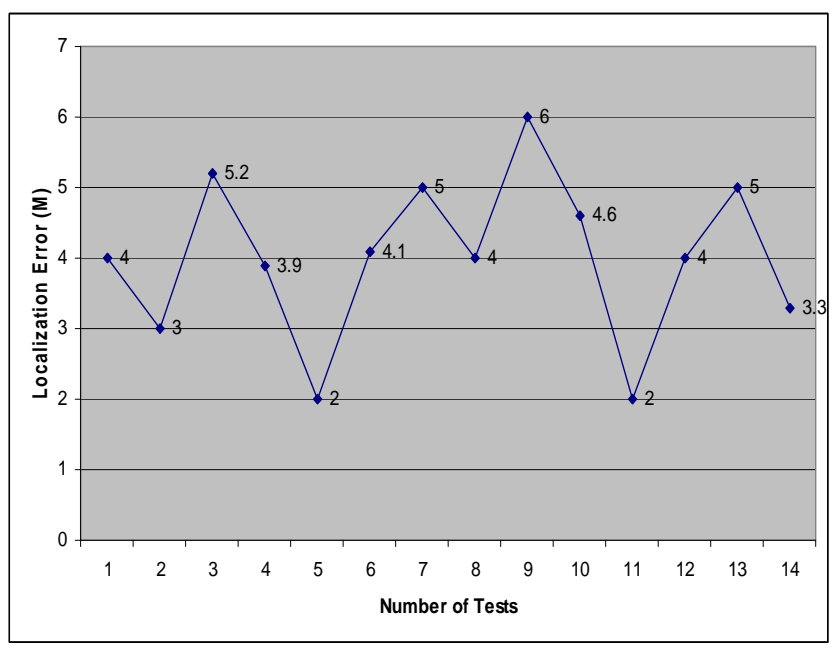

\subsection{Communication cost}

The communication cost can be calculated based on the number of messages which need to be exchanged among beacon nodes and the mobile target node. There are two main types of messages which need to be exchanged; firstly the messages which are exchanged between beacon nodes, and secondly, the messages which are exchanged between mobile nodes and beacon nodes. The average number of messages that are required to be exchanged is notated as $l$. Every time the mobile target enters a new area, it selects at least three beacon nodes in its range, and then the beacon nodes need to exchange a number of packets between them. Each beacon node sends at least three messages in order to find out the weight between beacon nodes. After that, every two seconds, each beacon node sends two beacon messages to the mobile target in its range. Consequently the average number of beacon messages which will be exchanged is implemented in equation 7.

$$
l=n \times f
$$

where $f$ is the final time, and $n$ refers to the total number of beacon nodes. $l$ is measured based on the number of beacon nodes $n$ which send two messages every two seconds to the mobile target. The beacon node should go back to sleep mode when the mobile target leaves the area. The overall cost is calculated based on the total number of messages over a tracking period of time.

The coordinator, which is a mobile target, is connected to a laptop which calculates and measures the position for each target node. This laptop will be replaced by a PDA in future.

\subsection{Comparison with previous works}

This section presents a comparison between our weighted RSS model and a normal RSS model. Both experiments were done in the same area, and under the same conditions to fix any variables affecting the results. The first step involves deploying a normal RSS model; readings were taken through 14 different points between the beacon nodes.

In the second step, readings were collected from the same points but using our weighted approach. We achieved a small localisation error (20\%) for $63 \%$ of all test points. Figure 9 shows the difference between these two models. The figure shows that the accuracy has been improved by deploying the weight function to the collected RSS values. This may give an indication about the effect of environmental characteristics on measuring the distance between mobile devices and beacon nodes. Apparently, localisation information is more correct and reliable when measured using a weighted RSS model than the conventional RSS model.

Figure 9 Weighted approach vs. normal RSS (see online version for colours)

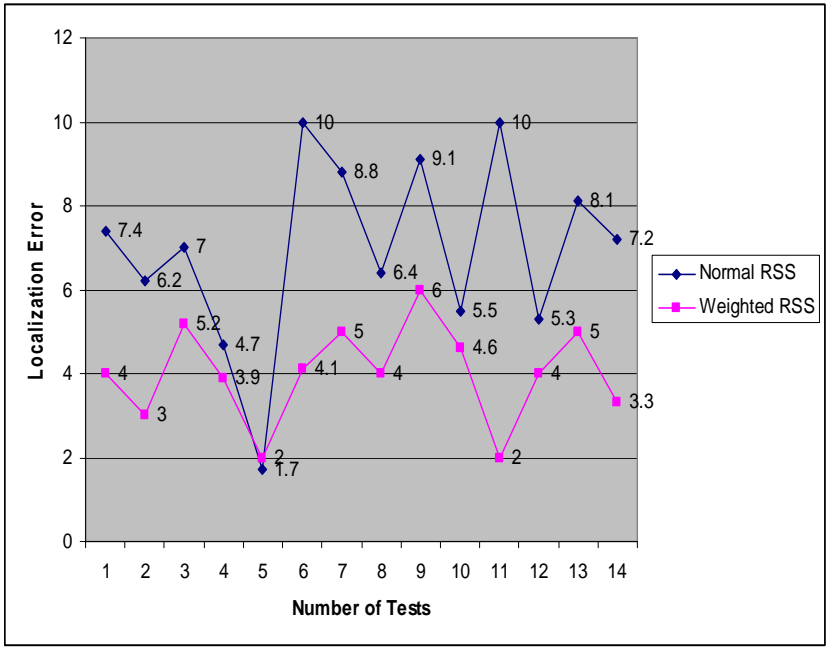

As mentioned above, there are many localisation techniques which use weights to improve localisation accuracy and efficiency. However, some of them suffer from requiring a specific spherical radio propagation assumption, and others can only be deployed in outdoors environments. This is clear in Bulusu et al. (2000) and Blumenthal et al. (2007), where highly accurate localisation information is obtained in an outdoors environment but their system can not work efficiently in noisy environments. Similarly, Blumenthal et al. (2005) and Reichenbach et al. (2006) involve localisation methods which assume that the transmission range is represented by a circle, which only can be applied outdoors.

The implementation of previous studies was limited in scale, as in Reichenbach et al. (2006), the experiments were implemented in a small area $(300 \times 300 \mathrm{~cm})$. The proposed work in this paper has been implemented in a realistic environment $(41.5 \times 11.3 \mathrm{~m})$. Our weighted approach is different from the previous presented work, as it's only based on the RF values and the distances between beacon nodes. The environment's characteristics were taken into consideration in order to improve the localisation accuracy. 
Figure 10 FK layout

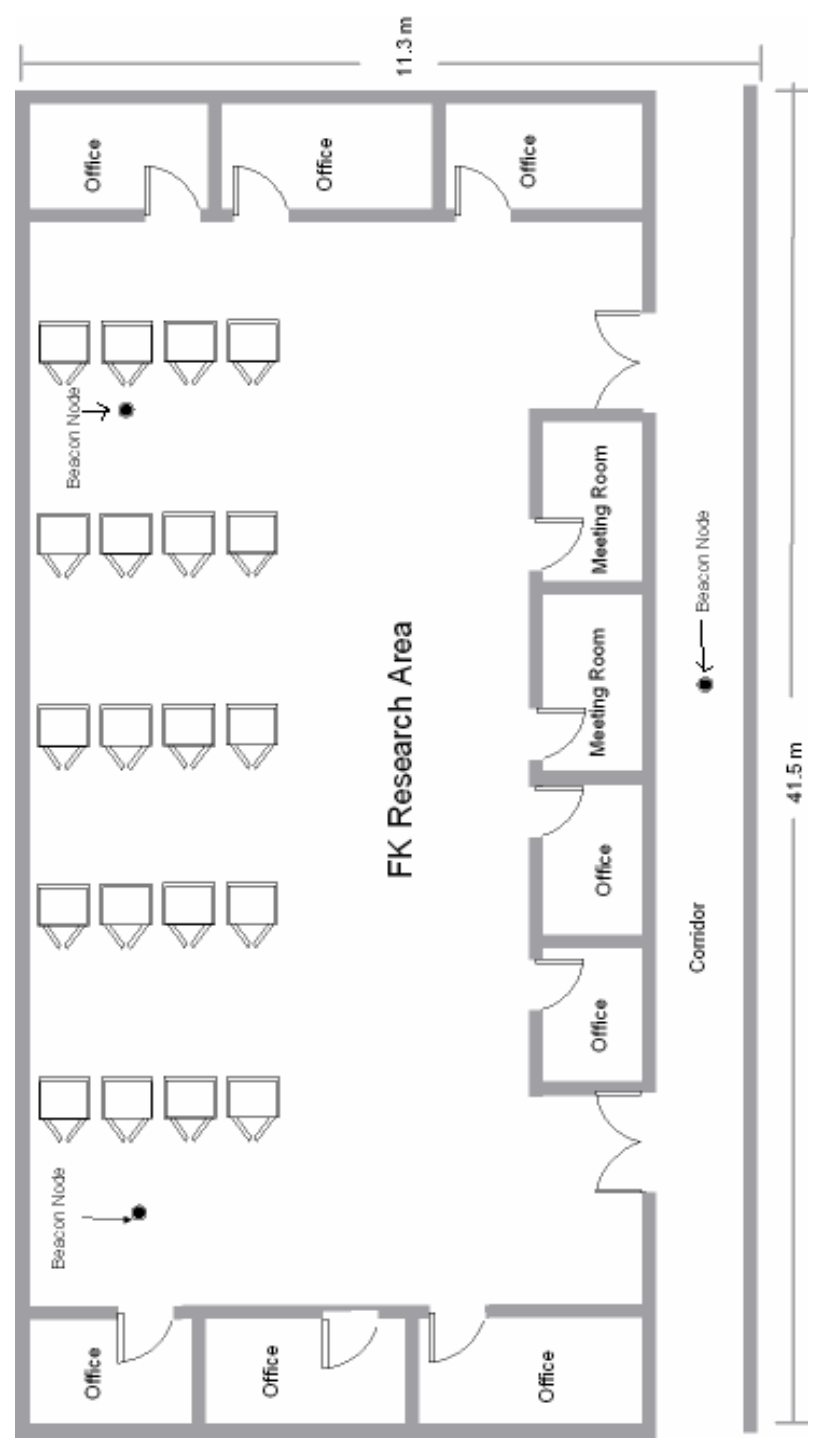

\section{Conclusions and future works}

Tracking mobile target applications through wireless sensor networks is a critical and emerging field. GPS is not the ideal system in indoors environments for two reasons: inaccessibility and expensiveness. ZigBee networks benefit from having the ability to quickly attach information, detach, and go to sleep mode, which offers low power-consumption and extended battery life. In this paper, we explored the RF-based localisation techniques, and other localisation techniques which are based on measuring the weights in order to calculate the position of the target nodes.

A new weight function is introduced, which is based on the distance between beacon nodes and the RSS values of them. The RSS system in combination with a weighted function offers lower communication overhead and lower localisation complexity. The localisation accuracy has been improved based on the collected weights from beacon nodes. The weight function can eliminate the effect of the environment's characteristics, and consequently helps in more accurately estimating the position for the target nodes.
One of the most important developments and improvements that should be added to research in the future is to improve the accuracy for our tracking system. The system should be deployed in larger area that could include a whole large building composed of several floors. This is important to test the efficiency and accuracy of the proposed system in a big building. Involving weights in this research potentially leads to greater accuracy. Investigating involving end-device nodes in the tracking process in order to track the mobile target when the density of router nodes is very low is one of the priorities in the future.

The proposed work involves tracking one mobile target. In the next step, we intend to track multiple mobile targets simultaneously, and increase the tracking area by involving a higher number of sensor nodes. A prediction technique should be deployed when the number of nodes is high, as some of these nodes should be on 'go to sleep' mode, when the mobile target is not in its range. Mobile targets will be looked at as possible routers in order to communicate with other routers in the ZigBee network.

\section{References}

Alhmiedat, T. and Yang, S. (2007) 'A survey: localization and tracking mobile targets through wireless sensor network', PGNet International Conference, ISBN: 1-9025-6016-7.

Blumenthal, J., Grossmann, R., Golatowski, F. and Timmermann, D. (2007) 'Weighted centroid localization in ZigBee-based sensor networks', Folien IEEE International Symposium on Intelligent Signal Processing, WISP, Madrid, Spain.

Blumenthal, J., Reichenbach, F. and Timmermann, D. (2005) 'Position estimation in ad-hoc wireless sensor networks with low complexity', Joint 2nd Workshop on Positioning, Navigation and Communication (WPNC 05) and 1st Ultra-Wideband Expert Talk, pp.41-49.

Brunato, M. and Battiti, R. (2005) 'Statistical learning theory for location fingerprinting in wireless LANs', Computer Networks, Vol. 47, pp.25-45.

Bulusu, N., Heidemann, J. and Estrin, D. (2000) 'GPS-less low-cost outdoor localization for very small devices', IEEE Personal Communications, Vol. 7, pp.28-34.

Rappaport, T. S. (1996) 'Wireless communications: principles and practice', Prentice-Hall Inc., New Jersey.

Reichenbach, F. and Timmermann, D. (2006) 'Indoor Localization with low complexity in wireless sensor networks', Proceeding of the IEEE (INDIN'06), pp.1018-1023.

Reichenbach, F., Blumenthal, J. and Timmermann, D. (2006) 'Improved precision of coarse grained localization in wireless sensor networks', 9th DSD Conference, Dubrovnik, Croatia, pp.630-637.

Roos, T., Myllymaki, P., Tirri, H., Misikangas, P. and Sievanen, J. (2002) 'A probabilistic approach to WLAN user location estimation', International Journal of Wireless Information Networks, Vol. 9, pp.55-64.

Shorey, R., Ananda, A., Chan, M. and Ooi, W. (2006) Mobile, Wireless and Sensor Networks, John Wiley \& Sons, Canada. 